\title{
Zur Frage der Spezialisierung der Geographie
}

Ernst Winkler

\section{Die Situation}

Die Entwicklung der Geographie kennzeichnet wie diejenige so gut wie jeder Wissenschaft fortschreitende Differenzierung und Spezialisierung, die sich in beinahe zahllosen Teil- oder Einzelgeographien ausdrücken. Dieser Vorgang ist, analog dem Prozeß der progressiven Aufgliederung der Wissenschaft überhaupt, eine Folge des sich fortwährend steigernden Strebens nach umfassender, detaillierter und vertiefter Erkenntnis. Er hat zur Arbeitsteilung in allen Bereichen des menschlichen Lebens und damit auch der Forschung geführt, die weiter anhält. Als Impulse dieser "Vervielfältigung» wirken die Erschließung "neuer» oder vorher unbekannter Gegenstandsbereiche (Tatsachen, Beziehungen, Gesichtspunkte) und die Entdeckung weiterer Verfahren, wobei auch Übertragungen von einer auf andere Disziplinen eine hervorragende Rolle spielen. Ein zwiespältiges Ergebnis dieses Prozesses ist zweifellos, daß vielfach der Überblick über das Ganze der wissenschaftlichen Arbeit verlorengeht, da der einzelne sich spezialisierende Wissenschafter zwar immer mehr von Teilen, dagegen je länger desto weniger von der Gesamtheit der Wirklichkeit zu erfassen vermag. Die dadurch bedingte Einseitigkeit erweckt den Eindruck der Zersplitterung der Wissenschaften, der man mindestens soviele Nach- wie Vorteile zuschreibt. Die Bestrebungen eines «Studium Generale» in verschiedenen Ländern nähren diese Befürchtungen besonders eindrücklich, während die "Empiriker» unter den Wissenschaftern ohne sonderliche Rührung zur Tagesordnung übergegangen sind, das heißt die Differenzierung den Erfordernissen eines wachsend tiefern Eindringens in das Sein gemäß unbekümmert weitertreiben.

Diese Situation fordert zur Prüfung heraus, die nicht allein erkenntnistheoretisch zu motivieren ist, sondern durchaus praktische Hintergründe hat. Insbesondere legt die Tatsache, daß die Geographie sich noch keineswegs jener durchgehenden Verwendung in der Praxis erfreut, die viele ihrer Vertreter für wünschenswert halten, eine Behandlung des Fragenkomplexes nahe. Sowenig dadurch eine tatsächliche Lösung zu erwarten ist, so entschieden kann eine solche möglicherweise ange- regt werden. An den Anfang des Versuches sei ein Zitat aus dem Buche "Die natürliche Ordnung der Wissenschaften» von Paul Oppenheim (1) gestellt. Es beginnt mit den Sätzen: «Der Erkenntnis von der Ordnung der Wissenschaften soll ein Bekenntnis vorausgehen: Die Wissenschaft ist ein lebendiges Ganzes. Das Bewußtsein von ihrer Ganzheit und Lebendigkeit ist unserer Zeit nur zu sehr verloren gegangen; unter der Herrschaft des Spezialistentums hat man den Überblick über das Wissensganze verloren; es wurde dem Leben entfremdet und durch die tote Summe nebeneinanderstehender Disziplinen ersetzt. Wo gegenseitige Anregung und gemeinsame Arbeit auf das gleiche Ziel hin herrschen sollte, trifft man auf intolerante Verachtung, auf mangelnden Kontakt, auf Kompetenzstreitigkeiten. Diesem Zustand entspricht das Chaos, welches der Student an den Universitäten antrifft, wenn er versucht, sich darüber zu orientieren, wie das Feld beschaffen ist, auf dem er sich betätigen soll. Aus all dem wird die tiefe Sehnsucht nach einer Ordnung der Wissenschaften erklärlich, welche den Übelstand beiseitigt». Natürlich werden zahlreiche Forscher - wie bereits angedeutet wurde - diese "Sehnsucht" in Frage stellen. Sie werden betonen, daß die Spezialisierung und Differenzierung notwendig sei, um den «Fortschritt der Erkenntnis» zu sichern. Daß dies durchaus der Fall ist, wird niemand bezweifeln oder bezweifeln können.

Auch in der Geographie wird der Differenzierungsbeziehungsweise Spezialisierungsprozeß weitergehen und weitergehen müssen. Denn einerseits ist das geographische Forschungsobjekt noch längst nicht in seiner vollen Realität und Komplexität erfaßt; andrerseits zeigt es, abgesehen von einem dauernden Wandel, immer wieder neue "Seiten", die «selbstverständlicherweise» zu fortwährender Vermehrung der Betrachtungsweisen nötigen. Die Frage ist lediglich, ob sich diese Differenzierung beliebig vollziehen kann und soll und vor allem auch, welches Verhältnis dabei zu den übrigen Wissenschaften und speziell zu den "verwandten" Disziplinen $\mathrm{zu}$ beachten ist. Um hierüber urteilen $\mathrm{zu}$ können, erscheint vorerst ein Überblick über die bisherige Entwicklung der Spezialisierung der Geographie angezeigt. 


\section{Bisherige Entwicklungen}

Ob die Geographie sich aus einer Wurzel oder aus mehreren entfaltet hat, beziehungsweise ob sie mono- oder polygenen Ursprungs ist, bleibe dabei dahingestellt. Eine der entscheidenden Phasen ihrer Geschichte, im Grunde eher eine Integrierungsals eine Differenzierungs- oder Spezialisationsphase war sicher, wie namentlich A. Hettner (2) dargelegt hat, ihre Erhebung aus einer vornehmlich angewandten, praktischen Disziplin, einer «Dienerin der Staatsverwaltung und der praktischen Interessen des täglichen Lebens» zur "reinen Wissenschaft». Sie ist besonders C. Ritter und A. v. Humboldt zu verdanken. Schon zu ihrer Zeit, in der ersten Hälfte des 19.Jahrhunderts stand dieser "Dualisierung» der Geographie eine "Pluralisierung» gegenüber. Sie findet etwa in der wesentlich von A. v. Humboldt begründeten Pflanzengeographie, in der Klimatologie, in der Morphologie (Geomorphologie) oder in der Kommerz- beziehungsweise Handelsgeographie und andern Zweigen Ausdruck. Dabei könnte wohl mit guten Gründen jede derselben weit in die Anfänge wissenschaftlicher Erkenntnis überhaupt zurückverfolgt und auch in den Rahmen anderer Wissenschaften (Botanik, Geologie, Wirtschaftswissenschaften) gestellt werden. $\mathrm{Ob}$ andrerseits gesagt werden darf, daß Wissenschaften wie Geophysik, Ozeanographie, Völkerkunde, Statistik und andere sich aus der Geographie entwickelt, aus ihr "gelöst» hätten, ist eine Frage, die sicher nur zu bejahen wäre, wenn der Begriff "Geographie» sich durch die historischen Epochen unverändert erhalten hätte, was kaum ohne weiteres anzunehmen ist.

Tatsächlich nahmen Differenzierung und Spezialisierung der Geographie wohl erst bewußtere Formen an, nachdem sie sich als Wissenschaft konsolidiert betrachten konnte, das heißt auf Grund ihrer Festigung als (mehr oder weniger) selbständiger Zweig der Erkenntnis, die, wie gesagt, auf Ritter und A. v. Humboldt zurückführt. Dieser Differenzierungsproze $\beta$ interessiert hier nur in seinen bisherigen Ergebnissen. Sie erweisen, daß er sich namentlich nach Sachkategorien vollzogen hat. Dabei unterlagen die beiden "alten» Hauptzweige: allgemeine und spezielle (besondere oder regionale) Geographie (auch Länderkunde genannt) verschiedenen Auslegungen. Je nach der Auffassung des betreffenden Vertreters läßt sich in diesem Zusammenhang von einer Zwei- oder Mehrgliederung in astronomische, physikalische (oder physische) und anthropische Geographie oder Anthropogeographie sprechen, die sich bekanntlich längst weiter in Geomorphologie, Klimatologie, Hydrographie oder Hydrologie (Grundwasser- und Quellenkunde, Potamologie, Limnologie, Ozeanographie, Glaziologie), Pflanzen- und Tiergeographie, Bevölke- rungs-, Sozial-, Wirtschafts-, Siedlungs-, Verkehrs-, Techno- und Staatengeographie (politische Geographie) und andere unterteilten. Doch hat die Differenzierung auch vor diesen Gliedern nicht haltgemacht. Um nur einige wenige «tertiäre» Zweige zu nennen, wurde die Wirtschaftsgeographie in Produktions-, Konsumtions- und Distributions- oder Handelsgeographie einer- und in Agrar-, Forst-, Fischerei-, Gartenbau-, Bergbau-, Energiewirtschafts-, Gewerbe- und Industriegeographie andererseits aufgetrennt, die ihrerseits schon wieder in neuer Zerfaserung begriffen sind. Die Siedlungsgeographie wiederum kennt Sonderungen in Hof-, Dorf- und Stadtgeographien. Dabei ist nicht immer ganz klar, ob es sich - wie bei andern Gebieten der Geographie - um Geographien sensu stricto oder mehr um geographische Sachdisziplinen, spezielle Geowissenschaften als Teildisziplinen systematischer Wissenschaften handelt, wie schon einzelne $\mathrm{Na}$ men: Hydrologie, Glaziologie, Klimatologie und andere andeuten.

Dies zeigt sich namentlich bei der Geomorphologie, die als Prototyp einer "physischen» Geographie oder Physiogeographie angesehen wird. Sie behandelt bekanntlich die Oberfläche der Erdrinde (Erdkruste, Lithosphäre oder auch, nach Ratzel, Geosphäre). Diese selbst ist Objekt der Geologie, welche sie sowohl nach ihrem "großstrukturellen» Bau (Tektonik) wie nach den Vorgängen in ihr (Dynamik), nach der Genese (historische Geologie, Stratigraphie) als auch regional (regionale Geologie) untersucht. Die Lithosphäre, beziehungsweise der "feste» Erdkörper, stellt aber auch Gegenstand der Geophysik, der Geochemie, der Petrographie, der Mineralogie (Kristallographie), der Pedologie und der Geomorphologie dar, wobei in der Regel nur letztere zur Geographie gerechnet wird. Auch sie hat sich übrigens in mehrere Äste verzweigt, von welchen - ohne Vollständigkeit zu beanspruchen - nur die Morphographie, Morphometrie, Fluvial-, Glazial- und Periglazial-, Wüsten-, Vulkan-, Gebirgs-, Litoral- und Karstmorphologie genannt seien. Bei dieser Vielfalt erhebt sich die - zwar nicht unbedingt hierher gehörige, aber doch für die Spezialisierung der Geographie schlechthin interessante - Frage, welche der in diesem Absatz erwähnten Disziplinen spezifisch geographische seien.

Was die Geomorphologie sensu lato als Lehre von den Formen der Erdkrustenoberfläche betrifft, gehört sie unzweifelhaft primär zur Wissenschaft von der Erdkruste selbst, insofern diese Oberflächenformen unmittelbar «Elemente» ihrer Gesamterscheinung sind. Sekundär erst, indirekt, als deren Element oder über sie hinweg, läßt sie sich logischerweise der Geographie einordnen. Dabei bleibt unbestritten, daß die Erdrinde eine wesentliche, ja eine basale Komponente des geographischen Objekts bildet. Indessen sind nicht nur ihre Oberflächenfor- 
men, sondern ebensosehr ihre Substanz, die Stoffe der Erdrinde für dieses Objekt von maßgeblicher Bedeutung; ihre Baugrund- und Nährgrundeigenschaften wie ihr Gehalt an Montanwirtschaftsrohstoffen beanspruchen für die Landschaft in keiner Weise geringere Wichtigkeit als das Erdrindenrelief. Damit aber wird die Geologie, beziehungsweise werden entsprechende Teildisziplinen der Erdkrustenlehre zu mindestens so integralen Gliedwissenschaften der Geographie wie die Geomorphologie, die wie gesagt bisher mit Ausnahme vielleicht der Bodenkunde, so gut wie ausschließlich dazu gerechnet wurde. Eine andere Frage ist, ob diese Disziplinen dieser Eingliederung zustimmen würden, da sie sich doch entschieden als selbständige Wissenszweige betrachten. Doch soll diese Frage hier nicht weiter erörtert werden. Sie führt uns jedoch zum maßgeblichen Problem, zur Frage (zurück), inwiefern sich eine Disziplin überhaupt spezialisieren soll und kann, damit sowohl die optimale Erkenntnis ihres Gegenstandes ermöglicht als auch ihr «Kompetenzbereich» im Interesse der wissenschaftlichen Arbeitsteilung gewahrt beziehungsweise nicht überschritten, oder, anders gewandt, bestmögliche Zusammenarbeit mit den übrigen relevanten Wissenschaften gewährleistet wird.

\section{Beurteilung}

Die Beantwortung dieser Frage hängt wohl unzweifelhaft sowohl vom Untersuchungsgegenstand wie auch vom Bestehen und vom Ausbildungsgrad der Wissenschaften ab, welche, als Nachbar- oder Hilfsdisziplinen, die Erfassung dieses Gegenstandes unterstützen. Was zunächst den spezifischen Gegenstand der Geographie betrifft, wäre unter anderem festzustellen, wie weit er ein (relativ) selbständiges, das heißt von andern unterscheidbares Gebilde repräsentiert, wo gegenüber andern Gebilden seine Grenzen liegen und welches die es konstituierenden, «arteigenen» Kriterien sind. Bezeichnen wir als Objekt der Geographie die Landschaft im Sinne des Gefüges von Litho-, Atmo-, Hydro-, Phyto-, Zoound Anthrosphäre (wobei dieses Gefüge durchaus statisch-dynamisch, individuell-nomothetisch, partiell-gesamtheitlich und sachlich-zeitlich-räumlich aufzufassen ist). Dann sind die ausschlaggebenden Fragen, wo es nach innen (das heißt in Richtung seiner Komponenten) wie nach außen (in Richtung einerseits des Erdinnern, andrerseits des Kosmos beziehungsweise des Sonnensystems) aufhört, das heißt durch andere Gebilde begrenzt ist, da an jenen Grenzen theoretisch (auch) andere Wissenschaften «beginnen». Im Blick hierauf könnte nun weiter gefragt werden: Bis wohin soll die Geographie in ihrer Differenzierung beziehungsweise Spezialisierung in Richtung ihrer Nachbardiszipli- nen gehen, um für sich selbst wie für diese anderen die optimale (rationelle, ökonomische) Arbeitsteilung $\mathrm{zu}$ sichern? Dabei sollen wünschenswerte Uberschneidungen hier nicht berücksichtigt werden.

Ein Beispiel aus dem Bereich der Pflanzengeographie, also aus einem Teilgebiet der Geographie, mag die Situation beleuchten. Diese geographische Teildisziplin wird nicht selten der Geobotanik gleichgesetzt. Mit A. Hettner (3), der sich seinerseits auf den Zoogeographen A. R. Wallace berief, darf indessen, im Interesse der Klarheit, ein grundsätzlicher Unterschied zwischen beiden gemacht werden. Die Geobotanik als ein Zweig der Botanik ist der Untersuchung der terrestrischen (oder auch landschaftlichen) Einflüsse auf die Pflanzen, auf ihre Individuen wie auf ihre Kollektive zugewendet. Die Pflanzengeographie hingegen hat, als Zweig der Geographie, umgekehrt die Wirkungen der Pflanzen auf die Landschaft zum Gegenstand. Bei der letzteren ist also die Pflanze "Element», Komponente eines andern Objekts beziehungsweise des Objekts einer andren Disziplin. Für die Geobotanik ist die Pflanze zentraler Gegenstand, die Landschaft "Element» oder besser beeinflussender Faktor desselben. Diese Unterscheidung der beiden Disziplinen erscheint eindeutig und einsichtig.

Die Frage indessen, die hier interessiert, ist, wie weit die Geographie als Landschaftsforschung in den Bereich der Botanik "hinein»gehen darf und soll. Hettner vertrat die Ansicht - und wandte sie bekanntlich auf alle Landschaftselemente: feste Erdoberfläche beziehungsweise Erdkruste, Gewässer, Lufthülle, Tiere, Mensch, Siedlung, Wirtschaft und andere an -, daß «die Geographie die Pflanzenwelt als Tatsachen der Erdräume und Erdstellen auf(zu)fassen" habe, während die Biologie die Pflanzen (und Tiere) als solche in ihren verschiedenen Eigenschaften, "wozu auch hier räumliches und zeitliches Auftreten gehöre», betrachte. Er fügte präzisierend hinzu, daß die "geographische» Verbreitung der Pflanzen und Tiere sowohl in die Botanik und Zoologie als auch in die Geographie eingehe, aber in verschiedener Weise: jener "sei es um die Pflanzen und Tiere (als ihrer Zentralobjekte), dieser um die Länder, Landschaften und Örtlichkeiten zu tun".

Damit erscheint die Grenze zwischen Pflanzengeographie (und Geographie) und Botanik (Geobotanik) eindeutig fixiert, wenn sich auch die Frage erheben mag (zum Beispiel bei O. Schlüter), ob in der Geographie nur die Vegetation oder auch Einzelpflanzen zu berücksichtigen seien. Auch sie dürfte indessen völlig positiv zu beantworten sein, insofern selbstverständlich auch Einzelpflanzen, zum Beispiel Bäume, Landschaften mitbestimmen können, namentlich in Steppen oder Wüsten, wo sie 
sogar physiognomisch besonders markant zu wirken vermögen. Doch müßte diese Frage im Grunde anders gestellt werden: nämlich inwiefern oder wie sehr der Geograph bei der Kennzeichnung einer Landschaft Einzelpflanzen zu berücksichtigen hat. Hier handelt es sich ohne Zweifel um ein «Maßstabsproblem», das heißt um eine Frage der detaillierteren oder weniger detaillierten Untersuchung und Darstellung der Landschaft.

Je einläßlicher, «differenzierter» eine Landschaft untersucht beziehungsweise dargestellt wird oder werden kann, desto umfassender werden auch ihre Elemente zu berücksichtigen sein. Doch wird in der Regel die (eigene) geographische Originaluntersuchung dort aufhören, wo (genügende) Ergebnisse der Nachbarwissenschaft, im vorliegenden Fall der Geobotanik oder Botanik schon vorhanden sind.

Um hier möglichst klar zu sehen, bedarf es einer näheren Erläuterung des Begriffs «Spezialisierung» (Spezialisation, die übrigens nicht unbedingt mit dem hier schon synonym verwendeten Terminus Differenzierung $\mathrm{zu}$ identifizieren ist). Spezialisierung bedeutet, auch in der Technik und in der Wirtschaft, Aus- oder Absonderung, Isolierung eines Phänomens aus einem meist übergeordneten (höhern) komplexeren und Beschränkung der Untersuchung auf dasselbe mit dem Zweck, es objektgemäßer (objektiver), das heißt: umfassender und zugleich differenzierter zu erkennen. So erstrebt zum Beispiel Spezialisierung in der Siedlungsgeographie auf den Hof, den Weiler oder das Dorf, beziehungsweise durch deren "Herausnahme» aus dem Gesamtsiedlungskomplex ihre genauere, detailliertere und damit wesensgemäßere Kenntnis - womit übrigens auch derjenigen der Siedlung schlechthin Förderung zuteil wird. Der Vorgang der Spezialisierung entspringt also weitgehend wenn nicht völlig dem Trieb nach bestmöglichem Begreifen sowohl der Teil-, als auch der Gesamtwirklichkeit. Nur geht - worauf anfangs hingewiesen wurde - beim Eindringen in die Details nicht allzuselten die Sicht aufs Ganze, im Beispiel der Siedlungsgeographie auf die Siedlungslandschaft oder die Landschaft als Ganzes verloren. Das Eindringen in «tiefere» Schichten eines Gegenstandes, in «Subelemente» von Elementen wird, anders ausgedrückt, oft mit einer Beeinträchtigung der Einsicht in das Gesamtgefüge erkauft, so paradox dies klingen mag.

Der Einwurf liegt nahe, ob Möglichkeiten einer Problemlösung überhaupt bestehen. Mit andern Worten, es frägt sich, ob im Blick auf ein bestimmtes Objekt, zum Beispiel eine Landschaft und damit im Rahmen der Geographie die Spezialisierung beliebig weit getrieben werden kann und muß, oder ob sich hinreichende Kriterien einer Einschränkung und Grenzziehung finden lassen, die sowohl das Ganze wie die Teile adäquat zu erkennen gestat- ten, ohne die Nachteile der Spezialisierung in Kaut nehmen zu müssen. Eine absolut eindeutige Antwort dürfce darauf kaum zu geben sein. Indessen liefern sowohl die Struktur der Gesamtwirklichkeit als auch die ihr längst angepaßte Gliederung der Wissenschaft in Teildisziplinen einleuchtende Anhaltspunkte dafür. Vor allem der Au bau der Welt aus wenn auch nicht völlig voneinander getrennten so doch deutlich unterscheidbaren Gebieden mannigfachen Komplexitätsgrades: vom Elementarpartikel über die Atome, Moleküle, Anorganismen (Kristalle, Minerale, Gesteine) und Organismen (Viren, Pflanzen, Tiere, Menschen sowie ihre Organe, Gewebe, Zellen), über Komplexe, die sowohl Lebewesen wie anorganische Komponenten enthalten (Landschaften, die Biosphäre s. 1.) und über die Gestirne und Gestirnsysteme bis zum Kosmos ist ein klares Indiz für eine "naturgeınäße» Spezialisierung der Wissenschaften. Ihr System bietet denn auch den durchaus passenden Schlüssel zur Frage, wie weit die Spezialisierung und insbesondere die Spezialisierung der Einzeldisziplinen zu gehen hat. Grundsätzlich wird sie - von einem höhern zu tieferen Gebilden oder von einem Ganzen zu Elementen und Subelementen - so weit fortschreiten, fortschreiten können und müssen, als nicht bereits, wie schon früher angedeutet, andere Disziplinen vorhanden sind, die sich mit diesen Gebilden hinreichend befassen. Daß dies auch für Gebildebeziehungen gilt, bedarf keiner besondern Erörterung.

Für die Geographie oder Landschaftsforschung bedeutet dies, daß sie sich primär auf ihr zentrales Objekt, die Landschaft im Sinne des schon erwähnten Gefüges von Litho-, Hydro-, Atmo- und Biusphäre zu konzentrieren, das heißt ausschließlich die Fragen zu beantworten hat, welche dieses Gefüge aufwirft. Die Komponenten desselben hat sie demnach einzig und allein so weit zu untersuchen, als sie das Gefüge selbst charakterisieren; die Hauptarbeit liegt also in der Erkenntnis ihrer spezifischen Korrelationen mit der Landschaft. Nun kann sogleich eingewendet werden, daß dieses Gefüge "Landschaft» in gewissem Sinne hierarchisch aufgebaut sei, aus komplexeren, einfacheren und elementaren, ja mikrokosmischen: molekularen, atomaren und sogar subatomaren Teilen beste. he. Hieraus resultiere die Frage, wie weit «hinunter» in diesem System die Geographie gehen dürfe und solle. Die Antwort hierauf kann nicht schwerfallen. Zunächst wird zu sagen sein, daß der Landschaftsforscher die Stufenfolge von einfachsten zu komplexeren Teilgebilden zum Ganzen der Landschaft selbstredend $\mathrm{zu}$ beachten hat. Andrerseits wird er in der eigenen Originaluntersuchung grundsätzlich nur bis zu jenen Stufen vordringen, bei welchen nicht schon Teil-, Hilfs- oder Nachbarwissenschaften ihm die notwendigen, hinreichen- 
den Teilerkenntnisse geliefert haben. Praktisch bedeutet dies, wiederum am Beispiel der natürlichen Pflanzendecke einer Landschaft erläutert, daß in der Regel zwar, je nach dem Detailliertheitsgrad der Untersuchung, sowohl die Vegetationseinheiten, also die Gesellschaftsklassen, -ordnungen, -verbände und -assoziationen als auch die Individuen zu berücksichtigen sind. Deren Erfassung ist indessen grundsätzlich Aufgabe des Botanikers beziehungsweise des Geobotanikers oder Pflanzensoziologen. Der Pflanzengeograph beziehungsweise Geograph hat nur ihre Anteilhaftigkeit an der Landschaft zu erkennen und darzustellen. Wo jene erstere Aufgabe freilich noch nicht gelöst ist, wird er sich ihr selbst unterz; shen müssen (und dies gilt bei jedem Landschaftselement), wobei er dann notwendigerweise zum Geobotaniker beziehungsweise Landschaftselementarforscher werden muß. Deswegen die Geobotanik der Geographie eingliedern zu wollen, liegt kein Grund vor. Sonst müßten, konsequent gedacht, dieser so gut wie alle übrigen Wissenschaften als Teildisziplinen subsummiert werden, was kaum wünschenswert erscheint (wenn auch andrerseits gesagt werden kann, daß jede Wissenschaft im Grunde in irgend einem Sinne Teildisziplin jeder andern ist). Die Spezialisierung der Geographie wird deshalb - um es nochmals zu wiederholen - im Bereich der Objektelemente prinzipiell dort am unanfechtbarsten aussetzen, wo noch unmittelbare Bezüge zum Gesamtgefüge zu erkennen sind. Daß dieser Grundsatz für alle Einzelwissenschaften Gültigkeit hat, bedarf gewiß keiner Begründung.

Es mag nun aber scheinen, als ob die Spezialisierungsmöglichkeiten der Geographie durch solche Argumentationen erheblich eingeengt würden. In Wirklichkeit sind sie keineswegs gering, wie anschließend kurz gezeigt werden kann. Dabei sei als Grundsatz festgehalten, da $ß$ diese Spezialisierung sich auf ihrem eigenen Bereich, auf der Ebene des Landschaftsganzen (Geochore, Geomer, Synergose, Geozönese, geographischer Aspekt und so weiter) vollzieht, möglichst nicht auf die Arbeitsfelder anderer Wissenschaften übergreift.

\section{Spezialisierungsrichtungen}

In mindestens drei bis vier Hauptrichtungen stehen der Landschaftsforschung Wege offen oder vielmehr: kann sie sich weiter konsequent differenzierend entfalten, um ihrem Gegenstande gerecht zu werden: nach Sachkategorien, Raum- und Zeitkategorien und in der Richtung von der Theorie zur Praxis.

In sachlicher Hinsicht läßt sie sich zunächst zweifellos noch erheblich weiter nach den Objekten Natur- und Kulturlandschaft spezialisieren als dies bisher der Fall war. Die Mannigfaltigkeit dieser Typen - denen beinahe ebensoviele Individuen entsprechen - ist so groß, daß der Forschung noch ein weites Betätigungsfeld bleibt, wobei die Untergliederung nach Landschaftsdominanten wie Relief-, Gewässer-, Vegetations-, Wirtschafts-, Siedlungslandschaften lediglich als grobe Andeutung erscheint. Nicht mit Unrecht haben in diesem Zusammenhang sowjetische Geographen entsprechende Vorschläge gemacht, so zum Beispiel A. G. Issaischenko (4), der in Anlehnung an die Biologie auf die Fruchtbarkeit der Spezialisierung nach Tundren-, Rentierweide-, polare Ackerbau- und Wüstenlandschaften hinwies. $\mathrm{Da} \beta$ in diesem $\mathrm{Zu}$ sammenhang besonders dem funktionellen Gesichtspunkt Aufmerksamkeit zu widmen wäre, braucht wohl kaum erwähnt zu werden. Lediglich anmerkungsweise sei betont, daß es sich bei den erwähnten Landschaftsbeispielen keineswegs um Geofaktorengebiete handelt; vielmehr sind darunter Landschaften zu verstehen, in welchen die genannten Geofaktoren ausschließlich Dominanten bilden.

Eine zweite nicht minder erfolgverheißende Spezialisierungsrichtung ist diejenige nach Raumkategorien. Ihre Leitidee steckt im Grunde bereits in Hettners Ansicht, die Geographie sei im Unterschied zu den Zeitwissenschaften und den systematischen Disziplinen (Natur- und Geisteswissenschaften) eine ausgesprochene Raumwissenschaft, und es erscheint geradezu merkwürdig, daß dieser Leitidee bisher nicht entsprechend nachgeeifert wurde. Indessen hat schon E. Obst (5) vor Jahrzehnten analoge Vorschläge vorgelegt, indem er die Schaffung von besondern Hochschul-Lehrstühlen für Erdteile und Meeresregionen sowie für Länder forderte. Und seither wurden verschiedentlich auch von andern Geographen ähnliche Anregungen gemacht, so jüngst von J. Schmithüsen (6) anläßlich des Rundgesprächs "Theorie der Geographie» in Saarbrücken, wobei ihm R. E. Dickinson und besonders V. Roglic unter Hinweis auf ähnliche Bestrebungen in der Sowjetunion sekundierten. Daß es sich hierbei sowohl um die Notwendigkeit handelt, verschiedene Dimensionen der Landschaft (von den Mikroregionen bis zur Globallandschaft) als auch Lagetypen zu berücksichtigen braucht hier nicht weiter verfolgt zu werden.

Beinahe unbeschränkte Möglichkeiten bietet weiter die "Zeitdimension». Die Periode ist vermutlich vorüber, da man die Geographie, nach der Ansicht von A. Hettner, als ausgesprochene oder sogar ausschließliche Gegenwartswissenschaft abstempelte. Abgesehen davon, daß der zeitliche Wandel der Landschaft als solcher vom Archaikum über die seitherigen Epochen und die Gegenwart bis in die Zukunft hinein zu verfolgen ist (nicht allein, weil dieser Wandel zu ihrem eigentlichsten Wesen ge- 
hört, sondern auch, weil ihm weder Historiker noch historische Geologen genügende Aufmerksamkeit schenken), bieten auch zeitliche "Querschnitte» als Basen des Verständnisses der Transformationen nicht minder wie als Objekte an sich und als Vergleichsobjekte unerschöpflichen Stoff zu gleicherweise interessanten wie wertvollen Untersuchungen. Es gibt so beispielsweise ebenso eine Geographie des geologischen Altertums, des Mittelalters, der Neuzeit wie zahlreiche Untersuchungen historischer Epochen wie etwa der Reformation, der Renaissance, der ersten industriellen Revolution, und nicht minder stellt die Zukunft, zum Beispiel von der Landschaftsplanung und -gestaltung her Aufgaben, welche der Landschaftsforscher auch zur eigenen geistigen Bereicherung lösen kann.

$\mathrm{Da}$ eine solche «dreidimensionale» Spezialisierung vielfache, naturgemäße Kontaktflächen besitzt, wobei sich räumliche und sachliche Untersuchungen mindestens ebenso häufig decken wie sie Inkongruenzen zeigen, tangiert die Wünschbarkeit ihrer separaten Inangriffnahme kaum entscheidend.

Aber die Wege der Spezialisierung sind damit noch keineswegs erschöpft. Wenn sie rückblickend betrachtet werden, muß auffallen, daß zwar im wesentlichen immer das Landschaftsganze im Zentrum blieb. Indessen hat dieses Ganze selbst verschiedene Seiten, die besonderer Beachtung würdig sind. Die Landschaften sind statische und dynamische Gestalten; in ihnen vollziehen sich "arteigene» Vorgänge, und sie stehen mit ihrer Umwelt, Nachbarlandschaften und fernern Gebieten, mit dem Kosmos und mit dem Erdinnern in vielfachen Beziehungen. Tauschen sie doch mit diesen Bereichen sowohl Stoffe als auch Energien aus, werden sie deshalb bald mehr oder weniger in einem quasistationären Gleichgewicht gehalten, bald schnellern oder langsamern Transformationen unterworfen und stellen sie schließlich - nach jeder dieser Wirklichkeits- und Betrachtungskategorie zahlreiche Individuen und Typen dar, die einer erkenntniskritischen Ordnung rufen. Einer Morphologie oder Strukturlehre der Landschaft(en) wären so in- und externe Funktionsdisziplinen (Physiologie, nach C. Ritter, A. Hettner, I. G. Granö; Oekologie oder Chorologie) und nicht zuletzt Transformationslehren (Landschaftsgeschichte, -genetik, -ontogenie und -phylogenie) sowie Systematiken (Taxonomie, Typologie) anzugliedern, die noch kaum entwickelt sind. So viele Namen, so weite Aussichten fruchtbarer Entfaltung, Vertiefung, Differenzierung!

Und schließlich zuletzt die Anwendung der Theorie der Landschaft auf das Leben, die Praxis: auf Landschaftsplanung, -gestaltung und -pflege und -nutzung. Auch hier öffnen sich verheißungsvollste Horizonte, wenn an ihnen auch nicht wenige Wolken ziehen. Fast paradox mutet dabei an, daß, wie bereits eingangs betont wurde, die Geographie bis ins 19. Jahrhundert hinein vorwiegend praktische Disziplin war, dann erst zur Wissenschaft erhoben wurde und nun erneut dem "Alltag» dienstbar gemacht werden soll. Diesem Vorhaben stehen jedoch nicht geringe Hindernisse entgegen; das hauptsächlichste liegt bei der Geographie selbst, die in den letzten Jahrzehnten zu sehr auf ihre "Wissenschaftlichkeit» gepocht hatte. Die Folge war, daß sie gerade bei jenen Stellen so gut wie unbeachtet oder besser: unbekannt - blieb, die für sie den wesentlichsten Einsatz hätten: bei der bereits vorhin erwähnten Landschaftsplanung (Orts-, Regionalund Landesplanung, Raumplanung, Raumordnung) und Landschaftsgestaltung. Die weitere, verhängnisvollere Folge aber war, daß inzwischen Disziplinen entstanden, die nicht allein, wie Landschaftspflege und Städteplanung sowie Städtebau, in Form von Grünplanung die Landschaftsplanung und -gestaltung annektierten, sondern bereits daran sind, von ihrer Tätigkeit aus auch die Landschaftsforschung in Angriff zu nehmen. Und wenn diese - wohl kaum bewußt gegen die Geographie gerichteten - Absichten auch noch keineswegs zur konsequenten Verwirklichung geführt haben, so könnte sich doch die Möglichkeit hieraus leicht ergeben, daß jene aus einer höchst wertvollen Praxis hinausmanövriert würde, bevor sie darin überhaupt Fuß gefaßt hat. In einigen Ländern glaubt man, dies durch Begründung von Hochschullehrstühlen für angewandte Geographie vermeiden zu können, besser: die Geographie mit dem Leben enger als bisher in Kontakt zu bringen. Es bleibt aber vorderhand eine Frage, wie dies tatsächlich bewerkstelligt werden kann. Denn in Wirklichkeit ist «angewandte Geographie» nicht mehr "Graphie»; sie ist, absolut gesehen: Anwendung der Geographie und das heißt: Landschaftsplanung und -pflege. Die erwähnten Lehrstühle müßten sich also vor allem um diese bemühen. Dazu gehört - wie immerhin vielen Geographen klar ist - sehr viel Technik, Oekonomie, Rechtswissenschaft, Sozialwissenschaft. Ästhetik, wobei die Einsicht in die Notwendigkeit prophylaktischer, konservierender, therapeutischer, regenerierender Maßnahmen entscheidend ist. Ob dies von geographischen Lehrstühlen aus geleistet werden kann und will, wird die Zukunft zeigen. Die Möglichkeiten, sich in gedeihlichster Weise zu betätigen, sind unbestritten. Die Durchsetzung entsprechender Absichten wird aber - in nur scheinbarem Kontrast zur erwähnten Spezialisierung - erhebliche Konzentration auf den eigensten Gegenstand der Geographie: die Landschaft oder das Gefüge der "Geosphären» verlangen, wozu nicht zuletzt die Abkehr von vielen Seitenwegen gehört, die andern Bereichen der Wissenschaft zustehen. Diese Ansicht ist keineswegs kassandrische Phantasie. Die Geographie hat au- 
Ber der Landschaft keine weitern Entfaltungsgebiete mehr; wohin immer sie, namentlich in Richtung der Landschaftselemente, vorstößt - nicht minder aber auch in Richtung außerlandschaftlicher Arbeitsfelder - , gerät sie in die Kompetenzräume anderer Wissenschaften, die dort nicht nur legitimere "Rechte» haben, sondern sie auch mit den Mitteln überlegener Techniken, Instrumentarien und wohl auch Finanzen zu wahren wissen. Die Landschaftsforschung hat also allen Grund, bei ihrem angestammten Objekt - übrigens eines der interessantesten, schönsten und für den Menschen wichtigsten, die sich eine Wissenschaft wünschen kann - zu bleiben und es ebenso forschend wie planend und gestaltend immer intensiver zu pflegen. Die Konzentration auf dieses Objekt wird ihr auch ihre Einheit als Disziplin erhalten, die im Blick sowohl auf ihre Eigenständigkeit als auch auf die Erkenntnis und Gestaltung der Landschaft dringend erwünscht ist. Dies hat schon F. Ratzel, einer der bewußtesten Förderer planvoller Spezialisierung, erkannt, als er - in seinem letzten großen Werk "Die Erde und das Leben» - schrieb: "(Die Geographie unterscheidet sich) von allen Nachbarwissenschaften durch ihren Umfang und die innere Verschiedenheit ihrer Gegenstände. Was sie aber als die eine Geographie zusammenhält, das ist ihre Aufgabe, die Erscheinungen der Erdoberfläche in allen Wechselbeziehungen zu erkennen, und ihre Methode genauer Beschreibung und umfassender Vergleichung in Wort, Karte und Bild».

\section{Rück- und Ausblick}

Zusammengefaßt darf - nochmals - betont werden, daß die Geographie zweifellos noch längst nicht am Ende ihrer Spezialisierung angelangt ist, sondern sie auch weiterhin, ja unablässig zu fördern hat, wenn sie als Disziplin Bestand haben will. Ebenso wichtig ist aber, daß diese Spezialisierung in ihrem eigenen Bereich erfolgt und nicht in Arbeitsfelder anderer Disziplinen eindringt, da sie sich dadurch nur selbst beeinträchtigt. Daß dies keinesfalls Isolierung, sondern im Gegenteil vermehrte Zusammenarbeit mit allen andern Wissenschaften bedeutet, bedarf keiner Erläuterung. Spezialisierung und Integrierung im eigenen Haus kann sie sich so nicht eindringlich genug zur Devise machen. $\mathrm{Zu}$ deren Vollzug kann als ein Pendant zum Eingangszitat ein Wort des Physikers A. Einstein als Impuls dienen, das er vor rund fünfzehn Jahren anläßlich eines Interviews mit der «New York Times» sprach: "Es ist nicht genug, den Menschen ein Spezialfach zu lehren. Dadurch wird er zwar zu einer Art benutzbarer Maschine, aber nicht zu einer vollwertigen Persönlichkeit. Es kommt darauf an, daß er ein lebendiges Gefühl dafür bekommt, was erstrebenswert ist. $\mathrm{Er}$ muß einen lebendigen Sinn dafür bekommen, was schön und was moralisch gut ist. Sonst gleicht er mit seiner spezialisierten Fachkenntnis mehr einem wohlabgerichteten Hund als einem harmonisch entwickelten Geschöpf.» Der Landschaftsforschung ist, wie wenigen Wissenszweigen von Natur aus anheimgegeben, beide Forderungen: die Spezialisierung wie die Integrierung aufs schönste zu erfüllen; es ist $z u$ hoffen, daß es ihr je länger desto überzeugender gelingen werde.

\section{Literaturhinweise}

1 Oppenheim, P.: Die natürliche Ordnung der Wissenschaften. Grundgesetze der vergleichenden Wissenschaftslehre. Jena 1926.

2 Hettner, A.: Die Geographie, ihre Geschichte, ihr Wesen und ihre Methoden. Breslau 1927.

3 Dasselbe wie (3).

4 Issatschenko, A. G.: Diskussionsvotum anläßlich der Sitzung der Abteilung für physische Geographie vom 12. Februar 1951, der Geographischen Gesellschaft der UdSSR.

5 Obst, E.: Eine neue Geographie. Die Neue Geographie. 1, 1922, H. 1, 4-13.

6 Schmithüsen, J.: Rundgespräch «Theorie der Geographie». Geographica Helvetica XXI, 1966, $36-37$.

\section{Specialization in Geography}

The present-day trend of scientific specialization is evident also in geography in its gradual partition into 'sub-geographies' (economic geography, urban geography etc.). The question arises as to the limits of such progressive specialization, and it is postulated that such limits exist there where the other sciences already concern themselves with the elements of the geographical study field, i. e. the region.

It is further shown that the direction of specialization required of geography, in particular with reference to the increasingly important applications in regional planning, lies in further research on integrated regional analysis rather than experimentation within fields of study better undertaken by other sciences (Economics, Geology etc.). 